\title{
Nanophotonic Lab-On-A-Chip Raman Sensors: a Sensitivity Comparison with Confocal Raman Microscope
}

\author{
Ashim Dhakal $^{1,2}$, Pieter Wuytens ${ }^{1,2,3}$, Frederic Peyskens ${ }^{1,2}$, Ananth Subramanian ${ }^{1,2}$, Andre Skirtach ${ }^{2,3}$, Nicolas Le \\ Thomas ${ }^{1,2}$, and Roel Baets ${ }^{1,2}$ \\ ${ }^{1}$ Photonics Research Group, Department of Information Technology, Ghent University-IMEC, Ghent (Belgium), \\ ${ }^{2}$ Center for Nano-and Biophotonics (NBphotonics), Ghent University, Ghent (Belgium), \\ ${ }^{3}$ Department of Molecular biotechnology, Ghent University, B-9000 Ghent (Belgium) \\ ashim.dhakal@intec.ugent.be
}

\begin{abstract}
- we compare the performance of the nano-photonic waveguide based evanescent Raman sensors and a typical the confocal microscope. For $1 \mathrm{~cm}$ long strip silicon nitride waveguides, we theoretically expect more than 500 times higher signal compared to the confocal microscopic systems. The results of our preliminary measurements indicate at least 50 times higher signal. For slotted waveguides, the preliminary measurement result yields about 300 times higher signal compared to confocal microscope while the theoretical figure is more than 2000.
\end{abstract}

Keywords-Integrated optics; Raman spectroscopy; Evanescent sensing

\section{INTRODUCTION}

Raman spectroscopy is a direct technique for detection and analysis of chemical and biological substances [1]. However, because of the extremely small cross section of the Raman scattering process, usually a high-power pump laser, sensitive detectors and longer detection time are required. This limits the widespread use of this valuable technique. While most of the existing Raman spectroscopy systems use a confocal microscope, a fiber probe or a hollow fiber, we recently proposed and demonstrated a lab-on-a-chip approach to Raman spectroscopy based on single mode nano-photonic waveguides [2]. In this approach, the molecules under study are evanescently excited and the corresponding Raman signal is also evanescently collected using the same single-mode photonic waveguides. This technique exploits the enhancement effects inherent to the high-index-contrast waveguide and a long interaction length, which as per our calculations, leads to at least two orders of magnitude higher signal than in confocal microscopes. Since a single waveguide carries the pump beam and also collects the Stokes light, it can be easily integrated on a chip with additional components, such as an Arrayed Waveguide Grating (AWGs) acting as an on-chip spectrometer, facilitating development of compact, low-cost systems with high performance [3]. Further, these nano-photonic chips can be mass-produced using CMOS-compatible process steps, which may lead to a disposable, point-of-need tool for Raman analysis of chemical and biological materials.
In section II, we briefly outline a theory of evanescent Raman sensing using single-mode photonic waveguides. In section III we outline a derivation of efficiency for confocal microscopes and show the theoretical results expected for silicon nitride waveguides. In sec IV we discuss the experimental setup and experimental results concerning the comparison of the measured Raman signal for photonic waveguides and that of confocal Raman microscopes.

\section{THEORY OF EVANASCENT RAMAN SENSING USING PHOTONIC WAVEGUIDES}

We quantify the efficiency of the waveguide by a parameter $\eta_{0}$ called conversion efficiency. $\eta_{0}$ is defined as the total scattered power $\left(P_{w g}\right)$ collected by a waveguide per unit length of the waveguide for a unit pump power $\left(P_{i n}\right)$ in the same waveguide when a random ensemble of the particles with unit scattering cross section $(\sigma)$ and unit concentration $(\rho)$ are distributed randomly and scattering incoherently in the vicinity of the waveguide $[2,4]$ :

$$
\eta_{0}\left(d z, \omega_{p}, \omega_{s}\right) \equiv \frac{1}{\rho \sigma\left(\omega_{p}, \omega_{s}\right) P_{p u m p}} \frac{d}{d z} P_{w g}\left(\omega_{s}\right)
$$

$\eta_{0}$ is a function of the electromagnetic mode as defined by the waveguide dielectric function. If the nanophotonic waveguide is invariant across its length, and the scattering particles lie uniformly in the upper cladding, then for any arbitrary length segment, $\eta_{0}$ is invariantly given by $[2,4]$ :

$$
\eta_{0}\left(\omega_{p}, \omega_{s}\right)=\frac{1}{n(\omega) k_{v}} \iint_{\text {up.clad }}\left(\frac{\pi}{\varepsilon_{0}} \frac{n_{g}(\omega) \lambda_{0}}{\tilde{A}_{e f f}\left(\vec{r}_{0}, \omega_{p}\right)}\right)^{2} d \vec{r}
$$

Here, the integral is carried out in the upper cladding region where the scattering particles are located; $\lambda_{0}$ is the excitation wavelength $n_{\mathrm{g}}$ is the group index of the mode $\varepsilon_{0}$ is the freespace permittivity $n$ is the refractive index of the medium where the scattering particles are located. $k_{v}=1.26 \times 10^{23} \mathrm{C}^{-}$ ${ }^{2} \mathrm{~V}^{2} \mathrm{~m}^{2}$ is a universal constant related to the fine structure constant characterizing the coupling between electronic charge and the electromagnetic field $[1,2,4] . A_{e f f}$ is defined as follows: 
$\tilde{A}_{e f f}\left(\vec{r}_{0}, \omega_{p}\right) \equiv \frac{\iint_{\infty} \varepsilon\left(\vec{r}, \omega_{p}\right)\left|\vec{e}_{m}\left(\vec{r}, \omega_{p}\right)\right|^{2} d \vec{r}}{\left|\vec{e}_{m}\left(\vec{r}_{0}, \omega_{p}\right)\right|^{2}}$
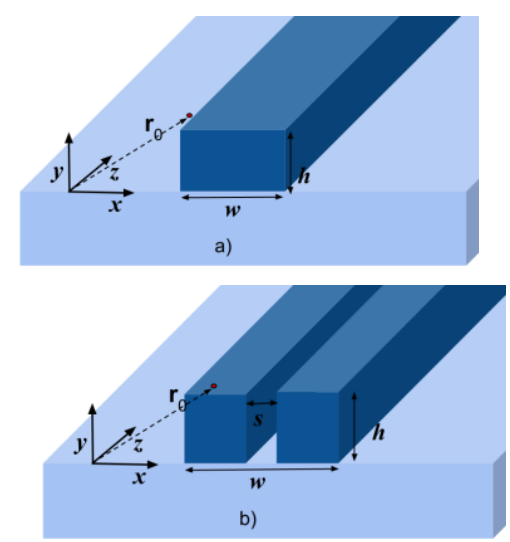

Fig. 1 a) Dielectric strip waveguide b) dielectric slot waveguides as typical examples of channel nanophotonic waveguides defined by a core of higher refractive index embedded on lower index bottom cladding (light blue, typically silicon oxide). The scattering particles are assumed to be embedded with a uniform density on the upper cladding (colorless region)

When deriving Eq. (2) we have assumed that [4]:

1. The waveguide core has a sufficiently small crosssectional area so that only fundamental guided modes exist.

2. The pump and stokes frequencies are sufficiently near, i.e. $\omega_{p} \approx \omega_{s} \approx \omega=2 \pi c / \lambda_{0}$, such that the corresponding group indices and the $A_{\text {eff }}$ are comparable.

3. There is only a radiative decay pathway and the power is low enough so that we are in a weak coupling regime and any saturation effects can be neglected.

4. We only consider off-resonance, non-degenerate adiabatic vibrational transitions corresponding to a pump frequency $\omega_{p}$ and a Stokes frequency $\omega_{s}$.

These are reasonable assumptions for scattering problems encountered in photonics.

\section{EFFICIENCY OF COLLECTION OF A CONFOCAL MICROSCOPE}

For diffraction limited confocal microscope, it is a reasonable assumption that only the particles located in the focal volume within the depth-of-focus $b$ contribute significantly to the signal. The excitation and collection efficiency can be defined as:

$\frac{P_{D L}}{P_{\text {pump }}}=\frac{\Omega}{4 \pi} \rho \sigma b$ where, $\Omega$ is the solid angle (corresponding to half angle $\theta$ ) subtended by the optics. The pre-factor $\Omega /(4 \pi)$ can be expressed in terms of numerical aperture $N A$ as follows:

$$
\frac{\Omega}{4 \pi}=\frac{1-\cos \theta}{2}=\frac{1-\sqrt{1-(N A / n)^{2}}}{2} \approx \frac{1}{4 n^{2}} N A^{2}
$$

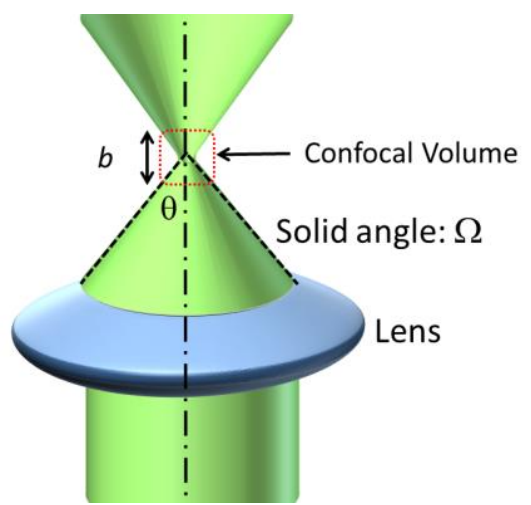

Fig 2. Schematic of the beam geometry at the objective (shown as a lens) for a confocal microscope. Only signal from confocal volume contributes to the signal.

We express the $N A$ and depth of focus $b$, for a Gaussian beam, $N A=\lambda_{0} /\left(\pi w_{0}\right), b=2 z_{R}=\pi n w_{0}{ }^{2} / \lambda_{0}$, then Eq. (4) reduces to:

$\frac{P_{D L}}{P_{\text {pump }}}=\frac{2 \lambda_{0}}{n} \rho \sigma$

Using Eq. (6) and Eq. (2) we can define a ratio $\Phi$ to compare the collection efficiency of diffraction-limited systems with efficiency of the lossless waveguides of length $l$ :

$\Phi=\frac{P_{w g}}{P_{D L}}=\frac{\eta_{0}}{2}\left(\frac{l}{\lambda_{0} / n}\right)$

$\Phi=\frac{\lambda_{0} l}{2 k_{v}} \iint_{\text {up.clad }} d \vec{r}\left(\frac{\pi}{\varepsilon_{0}} \frac{n_{g}(\omega)}{\tilde{A}_{\text {eff }}\left(\vec{r}_{0}, \omega_{p}\right)}\right)^{2}$

Fig. 3 shows the theoretical value of the enhancement ratio $\Phi$ calculated using Eq. (9) for $l=1 \mathrm{~cm}$ and $\lambda_{0}=785 \mathrm{~nm}$ with COMSOL finite elements mode solver. The simulation result shows that an enhancement factor of about 500 and 2000 can be expected respectively for typical strip and slotted waveguides.

\section{EXPERIMENTAL}

The measurement setup is similar to reported in [2], with an additional polarizer at the signal collection side so as to only collect light of a given polarization mode. For completeness, an illustration of the setup is also provided in Fig. 4. A tunable Ti-Sapphire CW laser was used as the pump source. For the experiments, a wavelength of $785 \mathrm{~nm}$ is coupled to the waveguide by end-fire coupling using an aspheric lens of effective focal length $8 \mathrm{~mm}(\mathrm{NA}=0.5)$. A halfwave-plate is used to control the polarization of the incident 
beam. The Raman signal (Stokes) collected by the waveguide that is co-propagating with the pump is then collimated via an achromatic objective $(50 \mathrm{x}, \mathrm{NA}=0.9)$ towards an edge filter, with the edge wavelength at $790 \mathrm{~nm}$ to block pump light going into the spectrometer. The Raman signal is then focused to a single-mode optical fibre (cutoff $=770 \mathrm{~nm}$ ) using a parabolic mirror of $15 \mathrm{~mm}$ effective focal length $(\mathrm{NA}=0.2)$ and

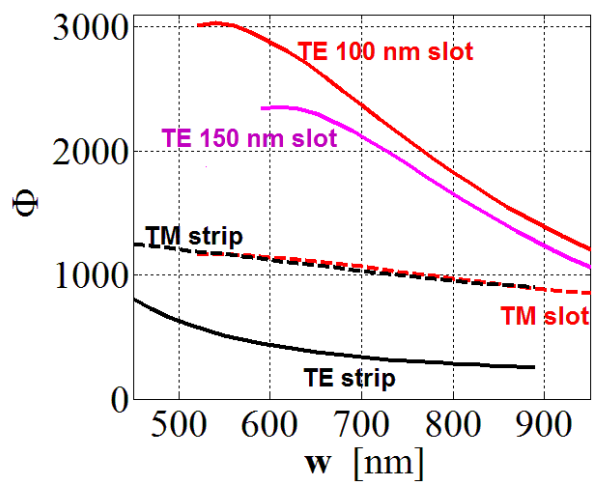

Fig 3. Theoretical values for the enhancement factor $\Phi$ compared to the confocal microscope as a function of waveguide width for $1 \mathrm{~cm}$ strip and slot silicon nitride waveguides and $\lambda_{0}=785 \mathrm{~nm}$. The values are calculated using Eq. 9 using COMSOL fine elements mode solver.

measured using a commercial spectrometer (AvaSpecULS2048XL). Because of the edge filter, the pump transmission is optimized and measured at $800 \mathrm{~nm}$ wavelength at the position of the spectrometer. The pump transmission at $800 \mathrm{~nm}$ is verified to be practically the same as for the pump wavelength $785 \mathrm{~nm}$ without the filter. The input laser power is set at $40 \mathrm{~mW}$, with an estimated $8 \pm 1 \mathrm{~dB}$ coupling loss per chip-facet. For experiments, strip waveguides of width $w=$ $550 \mathrm{~nm}$ and $w=700 \mathrm{~nm}$ are studied. Similarly, slot waveguides with slot width $s=150 \mathrm{~nm}$, and total waveguide width $w=700$ and $w=800$ are studied experimentally. The waveguides are wound as spirals (typical size: $800 \mu \mathrm{m}$ x 500 $\mu \mathrm{m})$ with bend radius $50 \mu \mathrm{m}$ and $150 \mu \mathrm{m}$ for strip waveguides and slot waveguides respectively so that the bend losses are negligible.

Pure Isopropyl Alcohol (IPA) droplets $\left(\rho=7.87 \times 10^{21}\right.$ molecules $/ \mathrm{cm}^{3}$ ) are applied on top of the waveguides as analyte to compare the Raman signal strength from waveguide and from the confocal microscope. We ensure that IPA covers the entire waveguide region of interest by visually monitoring the chip with a camera from top. The Raman spectra are extracted by fitting the background with truncated polynomials as explained in [2]. Fig. 5 shows a typical Raman spectra of IPA obtained using the procedure just described. We can clearly distinguish all the relevant peaks of IPA. The IPA peak corresponding to the C-C-O vibration at $819 \mathrm{~cm}^{-1}$ wavenumber ( $839 \mathrm{~nm}$, for $785 \mathrm{~nm}$ pump) is used as reference to compare the confocal system with the waveguide system. The total analog to digital converter (ADC) counts is converted to the corresponding power in units of watts by dividing the ADC counts with the corresponding integration time (typically 1-5 seconds) and the measured sensitivity $\left(19 \times 10^{6}\right.$ counts $\left./ \mathrm{ms} / \mu \mathrm{W}\right)$ of the spectrometer near the wavelength corresponding to the IPA peak. We take $\sigma=$ $7.9 \times 10^{-31} \mathrm{~cm}^{2} / \mathrm{sr}^{-1}$ for the $819 \mathrm{~cm}^{-1}$ line which is calculated using the value measured in [5] (measured using $488 \mathrm{~nm}$ Argon laser) and using the $1 / \lambda_{0}{ }^{4}$ dependence of the crosssection [1].

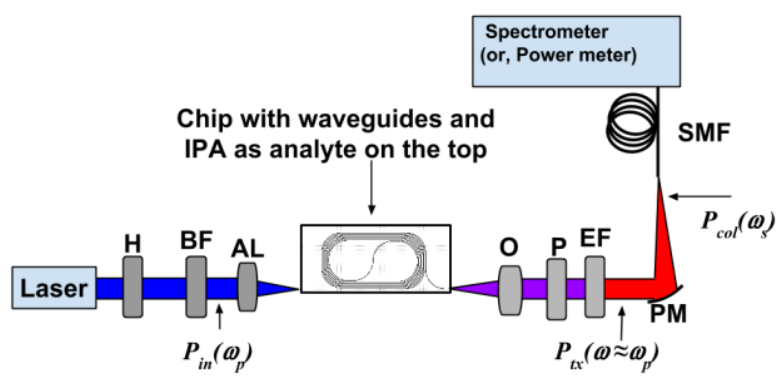

Fig 4. Schematic of the experimental setup to measure the Raman signal using photonic waveguides. H: half-wave plate, BF: Band-pass filter, AL: Aspheric lens. O: Objective, P: polarizer, EF: Long pass edge filter, PM: parabolic mirror, SMF: single-mode fiber. The chip and $A L$ are kept on a piezo-stage for precise in-coupling and out-coupling of light. Other symbols are explained in the text.

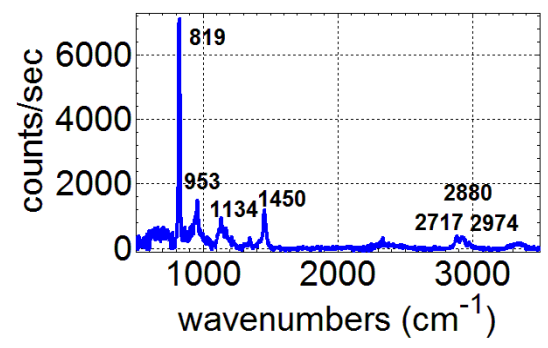

b)

Fig. 5. A typical spectrum of IPA measured evanescently after background correction.

As discussed in [2], to measure the conversion efficiency $\eta_{0}$ for several waveguides it is convenient to define a quantity $\zeta$ $(L)$ which is related to the quantities that can be measured directly:

$\zeta(L) \equiv \frac{P_{c o l}(L)}{P_{t x}}=\frac{1}{2} \rho \sigma \eta_{0}\left[\frac{e^{\Delta \alpha L}-1}{\Delta \alpha}\right]$

where, $P_{t x}$ is the transmitted pump power as measured by the power meter and $P_{c o l}$ is the scattered power collected by the waveguide that is measured at the spectrometer shown in Fig. 4. On the right hand side, we have added a factor of $1 / 2$ to account for the fact that we measure only the forward propagating signal. $\Delta \alpha=\alpha\left(\omega_{\mathrm{p}}\right)-\alpha\left(\omega_{\mathrm{s}}\right)$ is the difference between the waveguide losses $\alpha\left(\omega_{\mathrm{p}}\right), \alpha\left(\omega_{\mathrm{s}}\right)$ for pump and stokes frequencies respectively. The term in brackets evolves to $L$ when $\Delta \alpha \rightarrow 0$, as can be seen by expanding the exponent. The contribution due to the quantity $\Delta \alpha$ is generally small for small stokes shifts and shorter waveguide lengths.

The value of $\eta_{0}$ obtained for several widths for silicon nitride waveguide follows the trend predicted by Eq. (2) and agrees with the theoretical value [4]. This validates our theoretical 
model for channel waveguides. The experimentally estimated values of $\eta_{0}$ is close to $0.04 \mathrm{sr}$ for strip waveguides of $700 \mathrm{~nm}$ width and $0.25 \mathrm{sr}$ for $700 \mathrm{~nm}$ slot waveguides (inclusive of $150 \mathrm{~nm}$ slot width).

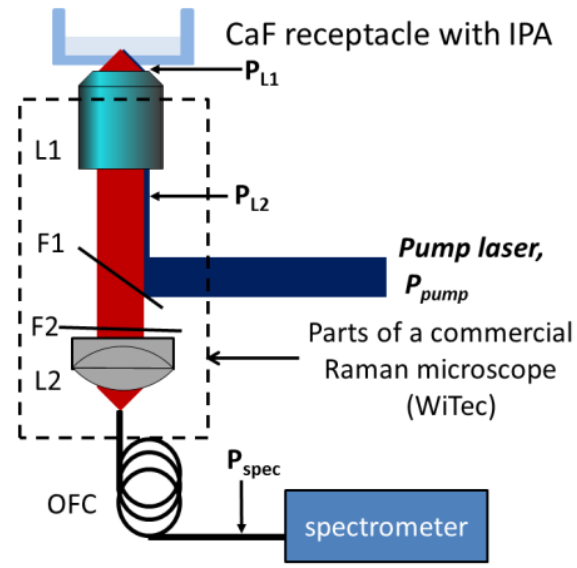

Fig 6. Schematic of the experimental setup to measure the Raman spectra using a commercial confocal microscope. L1: objective, F1: Dichroic filter reflecting the pump but transmitting the Stokes light, F2: Long pass edge filter, L2: lens to couple light into the multimode optical fiber cable (diameter $=100 \mu \mathrm{m}$ ), SMF: single-mode fiber. Other symbols are explained in the text.

The schematic of the experimental setup used for measuring the signal from confocal microscope is shown in Fig 5. The spectrum of IPA was measured in an inverted configuration so as to avoid artefacts that may result due to contact with the surface of the IPA and due to evaporation of the. IPA was contained in $\mathrm{a} \mathrm{CaF}_{2}$ receptacle with $0.7 \mathrm{~mm}$ bottom thickness. 10x Objective corrected for $0.7 \mathrm{~mm}$ cover thickness (Nikon CFI Plan Fluor 10x) was used for the measurement. Laser power at several locations of a commercial confocal microscope was measured to determine the transmission values for the objective, losses due to the filters and other optics in the microscope and efficiency of the fiber coupling in the confocal microscope. Pump power $P_{\text {pump }}$, power before the objective $P_{\mathrm{L} 1}$, pump after the objective $P_{\mathrm{L} 2}$ were measured. Further, the power coupled to the fiber $P_{\text {spec }}$ were measured by removing the filter $\mathrm{F} 1$ and $\mathrm{F} 2$ and sending a laser light directly from location L1 after removing the L1. The total estimated transmission was about $-11 \mathrm{~dB}$ from sample location to the MM fiber of $100 \mu \mathrm{m}$.

The measured signal power, normalized with the pump power at the location of sample and $\rho \sigma$ for the corresponding peak, was $5.5 \times 10^{-5} \mathrm{sr} \mathrm{cm}$. Taking all of the measured transmission and coupling losses $(-11 \mathrm{~dB})$ into account, we get about $7.5 \times 10^{-4} \mathrm{sr} \mathrm{cm}$. This is about 50 and 300 times smaller than the corresponding measured values of 0.04 and 0.25 for $1 \mathrm{~cm}$ waveguide. It is to be noted that our theoretical formula assumes a confocal volume defined by, $b=2 z_{R}=\pi n w_{0}^{2} / \lambda_{0}$. In our preliminary experiments, the confocal volume is much larger than that theoretically defined by $b=2 z_{R}$, as we use a $100 \mu \mathrm{m}$ multi-mode fiber as an aperture for practical reasons. This leads to the measurement of a larger signal for the confocal microscope.

\section{CONCLUSION}

We have compared the performance of nano-photonic waveguide based evanescent Raman sensors and the confocal microscope. For $1 \mathrm{~cm}$ long strip silicon nitride waveguides, we have theoretically calculated more than 500 times higher signal compared to the confocal microscopic systems. The results of our preliminary measurements indicate about 50 times higher signal for the strip waveguides. For slotted waveguides, the preliminary measurement result yields about 300 times higher signal compared to confocal microscope while the theoretical figure is more than 2000 .

\section{ACKNOWLEDGMENT}

The authors acknowledge the ERC advanced grant InSpectra for partial funding, and imec, Leuven for fabrication of the waveguides.

\section{REFERENCES}

[1] D. A. Long, 'The Raman Effect: A Unified Treatment of the Theory of Raman Scattering by Molecules', John Wiley \& Sons, Ltd, Chichester, UK (2002)

[2] A. Dhakal, A.Z. Subramanian, P. Wuytens, F. Peyskens, N. Le Thomas, R. Baets, "Evanescent excitation and collection of spontaneous Raman spectra using silicon nitride nanophotonic waveguides", Optics Letters, 39(13), 4025-4028 (2014).

[3] Ismail, N., L-P. Choo-Smith, K. Wörhoff, A. Driessen, A. C. Baclig, P. J. Caspers, G. J. Puppels, R. M. De Ridder, and M. Pollnau. "Raman spectroscopy with an integrated arrayed-waveguide grating." Optics letters 36, (23) 4629-4631 (2011).

[4] A. Dhakal, A. Raza, F. Peyskens, A.Z. Subramanian, N. Le Thomas, R. Baets, "Efficiency of evanescent excitation and collection of spontaneous Raman scattering near high index contrast channel waveguides" manuscript in preparation.

[5] M.J. Colles, J.E Griffiths, 'Relative and absolute Raman scattering cross sections in liquids', J. Chem. Phys., 7 (56) (2003). 